\title{
La qualité de l'aspect des placages de chênes : mesures de couleur et critères d'appréciation des professionnels français et italiens
}

\author{
JF Mazet, G Janin* \\ INRA, station de recherches sur la qualite des bois, centre de recherches forestieres, \\ Champenoux, 54280 Seichamps, France
}

(reçu le 17 février 1989 ; accepté le 2 août 1989)

Résumé - L'étude des différentes qualités possibles de l'aspect des placages de chêne (Quercus petraea (Matt) Liebl et Quercus robur $L$ ) et des critères qui permettent habituellement aux professionnels de les apprécier a été menée par une enquête (présentation de 53 paires de placage) auprès de 90 professionnels du bois français et italiens, répartis en 71 ateliers ou entreprises. L'objectivité de la démarche a été assurée par le traitement statistique des résultats (analyse factorielle des correspondances et tests du $\chi^{2}$ ) et par la caracterisation de l'aspect de tous les échantillons présentés par des mesures effectuées dans le système CIELAB de quantification des couleurs. II ressort des résultats que la différence de couleur (mesurable par $\Delta \mathrm{E}^{*}$ ) entre 2 placages favorise de manière nette la décision de choix.

La clarté des placages (mesurable par la luminance $L^{*}$ ) est une caractéristique appréciée d'une majorité de professionnels. Le caractère uniforme ou non des placages est également apparu important et bien décrit par la mesure de $s L^{*} / \mathrm{mL}^{*}$ (coefficient de variation de la clarté $L^{*}$ ). Enfin, la variabilité naturelle des tonalités de couleur (mesurables par l'angle de couleur moyen $h^{*}$ ) des placages europeens de chêne est largement suffisante pour générer des choix différents, spécifiques de certains groupes professionnels.

Quercus petraea / Quercus robur / bois de placage / analyse des correspondances multiples / couleur / mesure de qualité / critère de qualité

Summary - The quality of appearance of oak veneers: colour measurements and visual appreciation of French and Italian professionals. (Oak logs (Quercus robur $L$ and Quercus petraea (Matt) Liebl) are selected for the wood veneers' industry on the basis of their size, length, circumference, width and regularity of annual rings. After slicing, there are numerous quality indicators used to characterise the appearance of oakwood veneers (colour, figurations of wood, etc). The industrial classification of these different qualities is very complex, normally requiring the evaluation of several criteria. These criteria are descended from empirical and traditional use. The aim of this work was to clearly define these criteria in order to find the

* Correspondance et tirés à part 
best parameters to measure them, using the method of colour measurement CIELAB (1976), developed by the CIE (Commission Internationale de l'Eclairage). For this purpose, an inquiry was carried out, presenting to 90 French and Italian wood professionals a collection of samples of various industrial oak veneers $(20 \times 22 \mathrm{~cm}$ ), associated randomly in 53 pairs (fig 1). Each sample had been measured at 20 points using a spectrophotometer (Colorquest Hunterlab), which gives colour values in the CIELAB system. Thus, $L^{*}$ (the lightness), $h^{*}$ (the hue angle) and $s L^{*} / \mathrm{mL}^{*}$ (standard deviation for $L^{*}$, gained from the 20 measures) were obtained. For each pair, an opinion on quality was requested from each professional (preference for sample $A$, preference for sample $B$, no preference). It was not revealed to the professionals that the samples had been previously measured for their appearance. Multivariate analysis (Reciprocal Averaging) and $\chi^{2}$ tests, which were used for statistical treatment of data, clearly show that a great difference of colour between 2 veneer samples (measured by the total colour difference $\Delta E^{*}$ ), is an important factor in the choice of the professionals (fig 2). The first axis of the Reciprocal Averaging (fig 4), is connected with the uniformity of colour of the samples. The second axis shows that natural colour tone variability of European oak veneers is responsible for specific choices depending on different professional groups (fig 4). The standard deviation of the lightness $L^{*}$, and the hue angle $h^{*}$ appear to be related to these 2 professional criteria which are shown on the first 2 to axis of the analysis (figs 5 and 6). The lightness of oak veneers (closely associated with $L^{*}$, the lightness) is a very much appreciated characteristic for the majority of professionals (fig 11). In conclusion, it seems that it is possible to obtain some measured parameters which can objectively describe the qualities of the appearance of oak veneers.

\section{oak / veneers / multivarlate analysis / colour / colour measurement / criteria of quality}

\section{INTRODUCTION}

Les grumes de chêne (Quercus robur L et Quercus petraea (Matt) Liebl) sont habituellement sélectionnées pour le tranchage en fonction de leur longueur, de leur circonférence, de la taille et de la régularité de leurs accroissements annuels, et de l'absence de défauts graves de coloration. Une fois le tranchage effectué, la diversité de qualités des placages obtenus reste très grande. C'est pourquoi les entreprises de tranchage ont recours à des classements en qualité très complexes, comprenant souvent plusieurs dizaines de classes différentes. Pourtant, les critères de base de ces classements sont très difficiles à mettre à jour de manière objective, car ils sont souvent liés à une longue tradition de l'entreprise ou à un savoir-faire empirique et intuitif du personnel, qui éprouve de ce fait des dif- ficultés à les exprimer ; beaucoup de contradictions apparaissent en effet lorsque l'on discute comme nous l'avons fait avec des professionnels. II est en tout cas évident que ces critères reposent sur des caractéristiques d'aspect au sens large (régularité et tonalité de la couleur, degré de finesse du dessin, planéité, rugosité, présence ou absence de picots, de veines plus ou moins sombres, etc), caracteristiques qui conditionnent d'ailleurs souvent la facilité de mise en œuvre des placages (appariement, jointage, collage, ponçage, mise en teinte ou non, etc).

Afin de mieux comprendre et maîtriser la qualité de l'aspect des placages de chêne, une enquête sur échantillons de placages a été réalisée auprès d'un certain nombre de personnes concernées et les techniques spectrophotométriques de mesures de couleur (CIELAB) ont été utilisées. 
Les résultats concernant l'étude détaillee par analyse factorielle des correspondances des caractéristiques de qualité des placages de chêne spécifiquement recherchées par des groupes professionnels homogènes (fabricants de panneaux, par exemple) sont présentés dans un autre article (Mazet, 1989). Ce dernier inclut également l'analyse des réponses de quelques "non professionnels" du bois (en majorité des scientifiques). II ne fait en aucune façon intervenir les mesures de couleur.

L'objet du présent article est précisément de trouver des paramètres mesurables et donc objectifs (parmi ceux du système CIELAB) qui soient en relation avec les critères professionnels de qualification de l'aspect des placages, et qui aient donc un sens en terme de qualité.

Ces mesures, à l'usage éventuel de l'industriel, sont surtout destinées au forestier (gestion plus rationnelle de la ressource actuelle en chêne grâce à des mesures simples sur carottes de sondage) et au scientifique (études de variabilité géographique, comparaison de différentes espèces de chêne, etc).

\section{MATÉRIEL ET MÉHODES}

\section{Choix du type d'enquête}

Nous avons adopté un système souple et peu contraignant : la comparaison d'échantillons par paires.

Cette technique permet de multiplier le nombre d'échantillons présentés sans pour cela trop fatiguer le participant, la comparaison ne s'appliquant à chaque fois qu'à 2 échantillons sans qu'il soit nécessaire de se référer à tous les autres. La comparaison par couple d'échantillons est aussi la méthode utilisée par Brun-Chaize (1978) dans une enquête sur photographies concernant la perception des paysages forestiers par le grand public.

\section{Les échantillons de placages, sup- port de l'enquête}

Les échantillons présentés étaient des feuilles de placage de $0,6 \mathrm{~mm}$ d'épaisseur, provenant de grumes de chêne tranchées industriellement sur dosse et rive par les établissements Ober France. II s'agissait de chênes (Quercus petraea (Matt) Liebl et Quercus robur $L$ ) de provenances (française ou européenne) non précisées, la distinction entre Quercus robur et Quercus petraea n'étant pas faite puisqu'elle n'est jamais effectuee dans l'industrie, du fait qu'il est impossible à ce niveau de distinguer le bois de ces 2 especces.

\section{Constitution des couples de pla- cages}

En tout, 106 échantillons de placage ont été massicotés à $(20 \times 22 \mathrm{~cm})$ et répartis en 53 paires par tirage au sort. II convient d'insister sur cette constitution totalement aleatoire des couples : nous n'avons défini aucune raison possible de choix a priori (comme le dessin du bois ou la couleur) ; en effet, si nous avions associé les échantillons en fonction de telles ou telles différences précises qui nous paraissaient intéressantes, nous aurions pris le risque de faire réapparaître au cours du dépouillement statistique ces critères de choix qui étaient les nôtres sans forcément correspondre à ceux des individus interrogés.

Ces placages ont ensuite été fixés sur des supports en carton par de l'adhésif double face, permettant de conserver sla nervosite", les ondulations de certains placages. Pour la facilité des entretiens, les couples ont été reliés en livres, de manière à ce que 2 plaques d'un meme couple apparaissent jointives à l'ouverture (fig 1). L'ordre des couples dans chacun des livres a également été défini de manière aleatoire. En plus, à chaque entretien, nous avons effectué une rotation dans l'ordre de présentation des livres, pour pallier un éventuel effet de fatigue des personnes sollicitées. De cette manière, les premiers couples pré- 
sentés à certains entretiens se retrouvaient en dernière position à d'autres ; l'éventuel artefact dans les réponses, dú à la fatigue se trouvait ainsi réparti sur l'ensemble des couples.

\section{LIVRE 1}

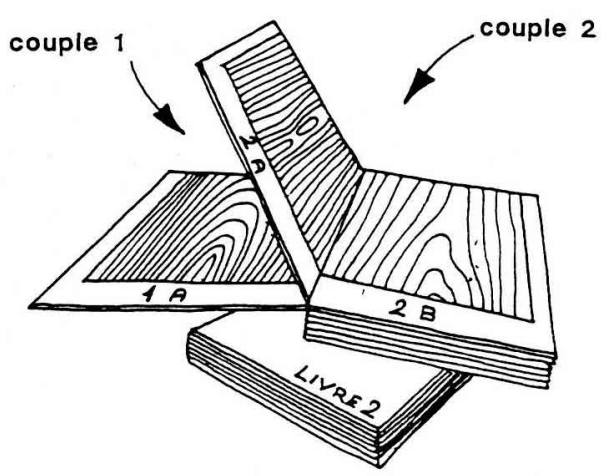

Fig 1. Forme sous laquelle ont été présentés les échantillons de placages de chêne.

\section{Mesures objectives effectuées sur les placages}

Les principes de la colorimétrie permettent de définir les coordonnées chromatiques d'un échantillon dans différents systèmes de couleurs dont le plus utilisé est celui proposé par la Commission Internationale de l'Éclairage en 1976 : CIELAB. De plus amples informations sur la mesure des couleurs et sur ce systeme en particulier figurent dans l'ouvrage de référence de Kowaliski (1978) et dans l'article de Janin (1987). Rappelonsen ici simplement l'essentiel : le système CIELAB est un système à 3 coordonnées de base $L^{*}, a^{*}$ et $b^{*}$ qui définissent un espace à 3 dimensions :

- $L^{*}$, la luminance, qui quantifie la clarté de l'échantillon (en $\%, 0 \%=$ noir, $100 \%=$ blanc), - $a^{\star}$, la coordonnée allant du vert $(-)$ au rouge $(+)$ (sans unité),

- $b^{*}$, la coordonnée allant du bleu $(-)$ au jaune $(+)$ (sans unité).

A partir de ces 2 dernières coordonnées peut être calculé $h^{*}$, l'angle de couleur (arc$\tan \left(b^{*} / a^{*}\right)$, en degrés, qui donne des informations quant à la tonalité de la couleur de l'échantillon: plutôt jaune (valeur d'angle éle- vee, proche de $90^{\circ}$, donc point de couleur proche de la partie positive de l'axe $b^{*}$ ) ou plutôt rose (valeur d'angle plus faible, donc point de couleur plus proche de la partie positive de l'axe $\left.a^{\star}\right)$.

Les valeurs de ces caractéristiques dépendent de la source lumineuse sous laquelle est observé l'objet. II en existe plusieurs, appelées illuminants et qui diffèrent par leur distribution spectrale d'énergie relative (voir Janin, 1987).

Dans notre cas, ces valeurs ont été obtenues pour l'illuminant de type D65 (caractérisant la lumière moyenne du jour) en utilisant un spectrophotometre ColorQuest Hunterlab à sphère d'intégration couplé à un micro-ordinateur IBM PC et pourvu d'une ouverture de mesure d'un diamètre de $9 \mathrm{~mm}$.

Afin de caractériser le plus objectivement possible l'aspect des échantillons présentés, 20 mesures réparties au hasard mais de manière à couvrir l'ensemble de la surface ont été réalisées pour chaque placage $(20 \times 22 \mathrm{~cm})$.

Sur chaque placage, les valeurs de clarté $L^{*}$ et des 2 coordonnées chromatiques $a^{*}$ et $b^{\star}$ ont été mesurées en 20 points.

Les caractéristiques suivantes sont alors obtenues :

- $m L^{*}$, moyenne des 20 mesures de clarté $L^{*}$;

- $s L^{*}$, écart type des 20 mesures de clarté $L^{*} ;$

- ma*, moyenne des 20 mesures sur la $1^{\text {ère }}$ coordonnée chromatique $a^{*}$;

- $m b^{*}$, moyenne des 20 mesures sur la $2^{e}$ coordonnée chromatique $b^{*}$;

- $h^{*}$, angle de couleur du placage (= arc$\left.\tan \left(m b^{*} / m a^{*}\right)\right)$;

- $s L^{*} / m L^{*}$, coefficient de variation de la clarté du placage.

De plus, le système CIELAB permet le calcul d'écarts de couleur entre 2 objets. Nous avons ainsi obtenu des indicateurs de la «distance visuelle» qui séparait les 2 placages $(A, B)$ de chaque couple :

$-\Delta E^{*}$, ecart de couleur $=\left(\left(m L^{*} A-m L^{*} B\right)^{2}+\right.$ $\left.\left(m a^{*} A-m a^{*} B\right)^{2}+\left(m b^{*} A-m b^{*} B\right)^{2}\right)^{1 / 2}$;

- $\Delta L^{*}$, ecart de clarté $=m L^{*} A-m L^{*} B$;

- $\Delta s L^{*} / m L^{*}$, écart d'uniformité d'aspect $=$ $\left(s L^{*} / m L^{*}\right)_{A}-\left(s L^{*} / m L^{*}\right)_{B}$;

- $\Delta h^{*}$, écart de tonalité de couleur $=h^{*} A^{-}$ $h^{\star}{ }_{B}$. 


\section{Population soumise à enquête}

L'enquête s'est déroulée en France durant septembre et octobre 1986 et en Italie en juillet 1987. Des professionnels du bois (90 en tout) ont été interrogés (entreprises de tranchage, fabrication de panneaux, de mobilier, entreprises d'agencement intérieur, menuisiers-ébénistes), dont 32 français et 58 italiens, représentant au total 71 entreprises ou ateliers.

Pour la France, les entreprises étaient réparties sur la région parisienne, le Jura et l'Est de la France. Pour l'Italie, nous avons essayé de cerner les environs de Florence ainsi que toute la région de la Brianza (Lissone, Seregno, Seveso. Meda), située au nord de Milan et qui est la région traditionnelle de tranchage.

L'Italie a été choisie comme exemple de pays étranger pour des raisons de langue. d'opportunité et d'importance économique pour ce secteur. La France est son premier fournisseur de grumes de chêne. L'Italie représente en effet notre $3^{\theta}$ marché dans ce secteur après celui de la RFA (Inf Bois, 1985 et 1987). De plus, les exportations de placages français, qui représentent 60 à $70 \%$ de la production sont effectuées en quasi-totalité vers la RFA et l'italie (Rev Bois, 1985).

\section{Le questionnaire; déroulement d'un entretien}

Les placages ont toujours été présentés à la lumiere du jour.

Le questionnaire était d'administration directe, c'est-à-dire que les questions étaient posées directement par l'enquêteur qui inscrivait également lui-me̊me les réponses sur un bordereau.

Il étaî́ simplement demandé à la personne interrogée d'effectuer un choix entre la plaque $A$ et la plaque $B$ de chaque couple, l'acceptation ou le rejet des 2 étant également possible (non-choix). Les remarques ou les éventuelles justifications spontanées de choix étaient également prises en compte par écrit. A l'origine, le questionnaire comprenait une partie réservée aux raisons du choix, qui devaient obligatoirement etre énoncées parmi les 3 suivantes : la couleur, le dessin et la rugosité. Cette formule a été rapidement abandonnée car dans de nombreux cas, il était impossible aux gens de justifier leur choix. En plus, nous avons pu maintes fois constater que, comme le souligne Javeau (1985), *... ce type de questionnaire fermé présente parfois le danger de dicter la réponse à la personne dans la mesure où, n'autorisant pratiquement aucune expression de nuances, il risque d'aiguiller celle-ci vers la réponse qui lui semble, non pas la plus proche de ce qu'elle pense et qui d'ailleurs peut ne pas figurer dans les choix possibles, mais la plus conforme à l'attente des réalisateurs d'enquête..... D'autre part, l'analyse statistique utilisée ne nécessitait pas cette partie du questionnaire, qui ne faisait qu'alourdir l'entretien.

Le temps de présentation des couples variait de 20 à $35 \mathrm{~min}$.

Au moment de leur choix, les individus interrogés n'avaient pas connaissance des différentes mesures de couleur effectuées sur les placages.

\section{RÉSULTATS}

\section{Mise en évidence du róle de la cou- leur dans les choix en qualité}

Dans un premier temps, il etait intéressant de chercher à savoir de manière objective si la couleur est effectivement prise en compte dans les choix en qualité effectués par des professionnels. Le paramètre $\Delta E^{*}$ a donc été utilisé car il représente la différence totale de couleur existant entre 2 objets. En effet, il prend en compte aussi bien la difference de clarté que celle de tonalité.

L'enquête une fois réalisée, les nombres de réponses "choix" et "non choix" ont été comptabilisés, après avoir répartis les 53 couples en fonction de leur valeur de $\Delta E^{*}$ (classes de $\Delta E^{*}, 0-2 / 2-3 / 3-$ $4 / 4-6 / 6-11 /, 15$ couples entrant dans la classe $0-2 /$, etc).

Un tableau de contingence a alors été construit sur lequel le test du $\chi^{2}$ a été réalisé ; les résultats sont présentés ici sous la forme d'un histogramme relatif aux "non-choix" (fig 2). Dans ce 
cas particulier, le test nous permet de savoir si le degré d'écart en couleur, mesuré entre les 2 plaques d'un couple, a influencé ou non le nombre des réponses "non-choix" à ce couple.

Nous constatons qu'il n'y a pas d'indépendance entre le degré d'écart en couleur $\Delta E^{*}$ des couples et le nombre de choix ou de non-choix effectués. En d'autres termes, il semble qu'il y ait une tendance générale à choisir plus facilement lorsque les 2 placages sont bien différents en couleur. Nous vérifions ainsi que la couleur joue donc un

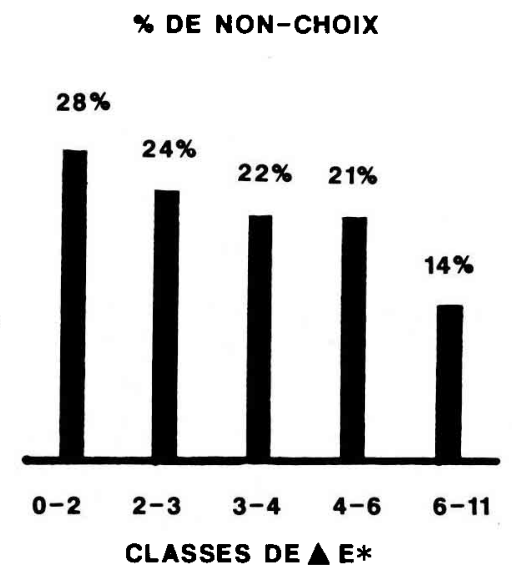

Fig 2. Influence de l'écart total en couleur entre les deux placages sur le nombre de non-choix; (analyse des choix de 90 professionnęls du bois aux 53 couples de placages). Test du $\chi^{2}$ significatif $(1 \%)$ : les classes different significativement entre elles.

rôle indéniable dans l'appréciation professionnelle de la qualité de l'aspect des placages de chêne.

\section{Analyse du rôle de la couleur dans les choix en qualité}

\section{Analyse factorielle des correspon- dances sur les données}

\section{Codage des données}

Nous avons appliqué un codage logique disjonctif complet (en 0 et 1 ) aux grilles de réponses obtenues à la suite de l'enquête pour pouvoir les soumettre à l'analyse statistique. Le choix de la plaque $A$ ou $B$ dans un couple a été codé sur 3 colonnes respectivement par (100) ou (010), tandis qu'une hésitation ou un non-choix ont été codés par (001). Pour caracteriser les individus interrogés, nous avons utilisé 2 variables illustratives codées l'une sur 10 colonnes (catégories professionnelles) et l'autre sur 2 colonnes (pays).

Présentation de l'analyse factorielle des correspondances (AFC)

On peut trouver une présentation détaillée de cette méthode dans les ouvrages de référence (Benzecri, 1980 ; Lebart et Fenelon, 1973). Pour résumer l'essentiel, rappelons que I'AFC facilite la mise en correspondance des individus et de leurs réponses (représentation simultanée fondée sur le principe barycentrique).

Le logiciel utilisé était ANALY de la programmathèque statistique AMANCE 81 (Bachacou et al 1981).

\section{Résultats}

Nous rappelons que l'analyse détaillée des donnees par AFC, sans tenir compte des mesures, (6 analyses effectuees sur differents sous-tableaux) est présentée dans l'article de Mazet (1989). Nous présenterons donc ici les résultats de l'AFC d'un tableau de (90 lignes $\times 106$ colonnes) (90 professionnels du bois et leurs choix relatifs aux 53 couples de placages).

L'analyse nous fournit les pourcentages d'inertie expliquée par chacun des 7 axes extraits (fig 3). Dans le cas de données en 0 et 1 , ces valeurs sont toujours très faibles et il ne faut s'intéresser qu'à leurs écarts relatifs qui permettent de connaître le nombre d'axes susceptibles d'être in- 
$\operatorname{axes}$
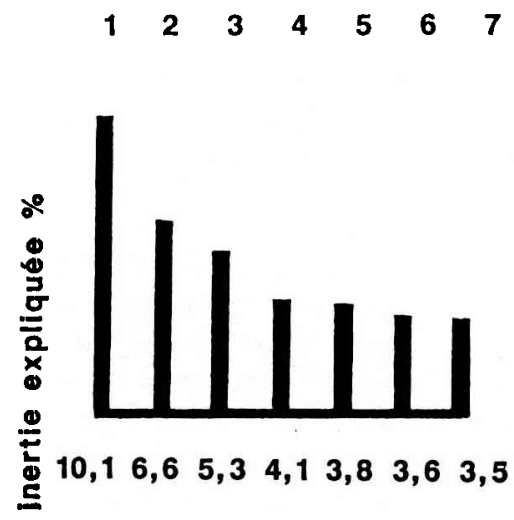

Fig 3. Pourcentage d'inertie expliquée par chacun des 7 axes extraits; (AFC sur les choix en qualité de 90 professionnels du bois aux 53 couples de placages).

terprétés ; un écart important entre 2 axes consécutifs traduit une diminution de la quantité d'information susceptible d'être apportée par le $2^{e}$ axe. II apparaît ici que les 3 premiers axes sont susceptibles d'être interprétés.

Recherche des principaux critères de qualité de l'aspect des placages

La partie gauche de l'axe 1 (fig 4) est représentée par le choix de 12 plaques qui s'opposent à la moyenne des choix (centre du graphique, intersection des 2 axes); en observant après coup ces échantillons, il apparaît clairement qu'ils ont tous en commun un aspect non uniforme, taché, veiné ou des plages de vaisseaux importantes. Le "déséquilibre" de cet

\section{AXE 2}

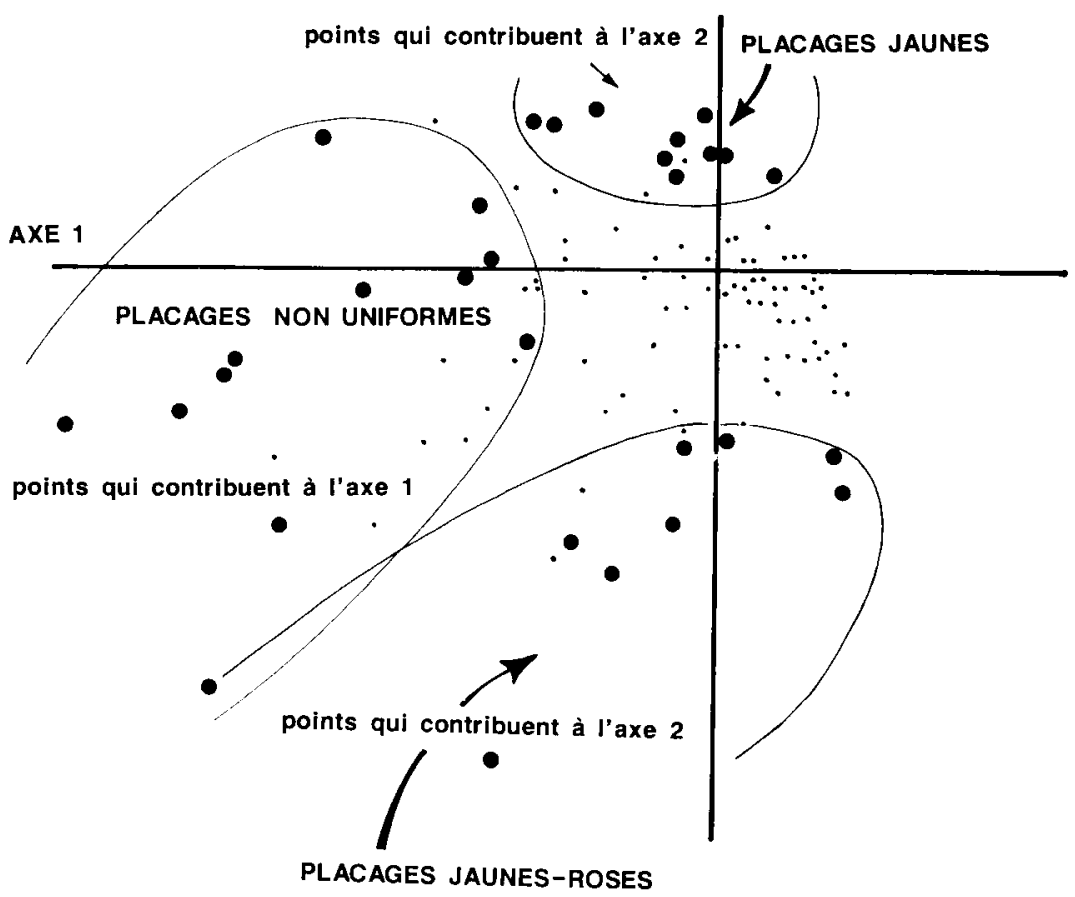

PLACAGes JAUNES-ROSES

- projections des choix de placages ayant fortement contribué à la formation des axes - projections des choix de placages

Fig 4. Projection sur le premier plan fourni par l'AFC des choix de placages qui ont le plus contribué à la formation des axes; (AFC sur les choix en qualité de 90 professionnels du bois aux 53 couples de placages). 
axe indique que ces placages ne sont appréciés que par une minorité d'individus. Ces individus s'opposent ainsi, par leurs choix, à l'ensemble des autres, qui apprécie plutôt des placages d'aspect uniforme et régulier. En tout cas, un critère lié à l'uniformité de l'aspect et de la couleur des placages de chêne est donc utilisé par les professionnels.

Une observation des échantillons nous indique que l'axe 2 de la figure 4 oppose manifestement des choix de placages plus rosés (bas de l'axe) à des choix de placages plus jaunes (haut de l'axe) par rapport à ceux auxquels ils étaient comparés . Ici, contrairement au cas précédents, l'axe possède 2 pôles de part et d'autre du centre : les choix sont donc répartis de manière equilibrée entre 2 tendances. La variabilité naturelle des tonalités de couleur chez le chêne européen (Quercus robur $L$ et $Q$. petraea (Matt) Liebl) est donc tout a fait suffisante pour générer des choix différents de la part des professionnels concernés.

Du fait de la nature de ces 2 critères mis en évidence, (uniformité et tonalité de la couleur), il est naturel de penser qu'ils pourraient correspondre aux mesures de $s L^{\star} / m L^{*}$ et $h^{\star}$. Cela se vérifie : - lorsqu'un repérage des échantillons est effectué sur ce premier plan fourni par l'AFC en utilisant les valeurs mesurées du coefficient de variation de la clarté $s L^{*} / m L^{*}$ (fig 5), force est de constater que les placages qui possè-

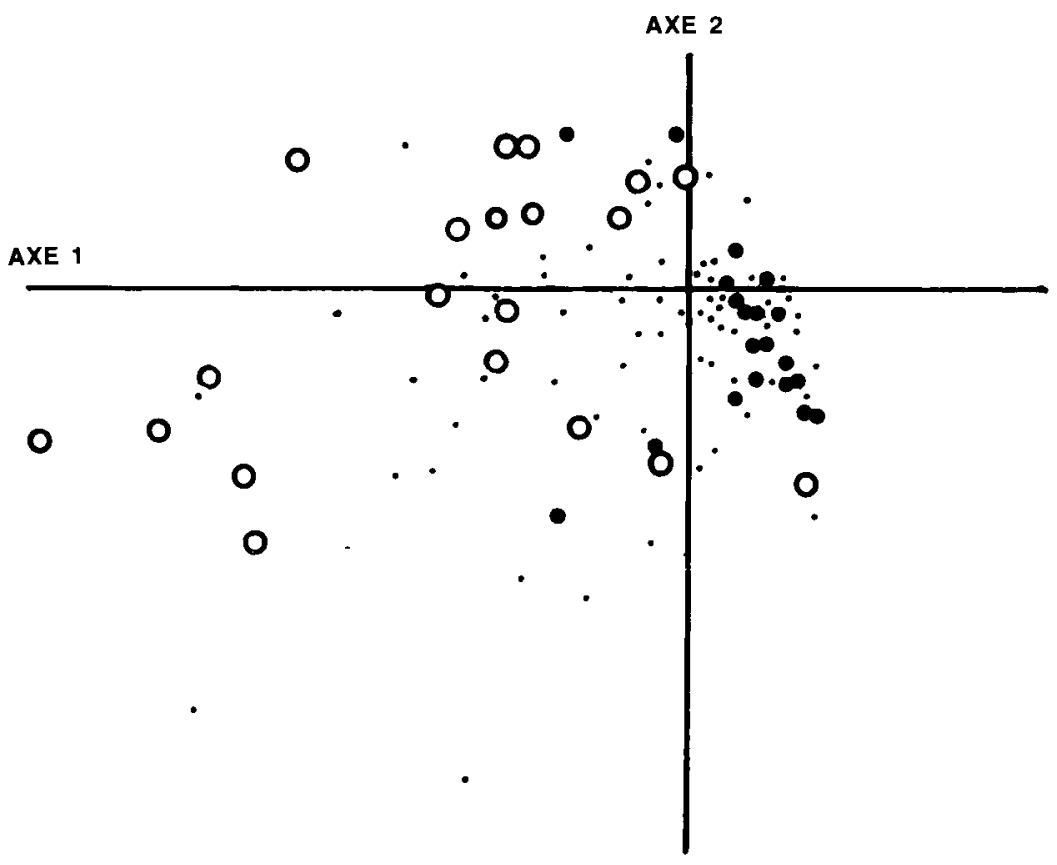

couples de placages avec écart $\Delta \mathrm{s} L / \mathrm{mL}$ supérieur à 1

$O$ placages avec valeur de $\mathrm{sL} / \mathrm{mL}$ forte

- placages avec valeur de $\mathrm{sL} / \mathrm{mL}$ faible

- projections des autres placages

Fig 5. Recherche d'adéquation entre la mesure de $S L^{*} / m L^{*}$ et le critère d'uniformité dégagé par l'axe 1 (AFC sur les choix en qualité de 90 professionnels du bois aux 53 couples de placages). 
dent de fortes valeurs pour ce paramètre sont situés dans la partie gauche du plan, et inversement (seuls ont eté pris en compte les couples pour lesquels l'écart $\Delta s L^{*} / m L^{*}$ entre les 2 placages était $\geq 1$ ). Le coefficient de variation de la luminance $s L^{*} / m L^{*}$ apparaît donc être en effet un indicateur objectif de la qualité de l'apparence des placages de chêne, puisque traduisant un critère professionnel.

- si l'on s'intéresse maintenant aux couples de placages pour lesquels l'écart mesuré $\Delta h^{*}$ est $>2$, et que l'on repère, toujours sur ce premier plan de l'AFC, les placages qui possèdent la valeur de $h^{\star}$ la plus élevée et ceux qui possèdent la valeur la moins élevée du couple, on obtient la répartition de la figure 6. La séparation sur l'axe 2 de l'AFC est bonne, à 2 exceptions près. Ce résultat confirme notre interprétation de l'axe en terme de tonalité de couleur. II indique aussi et surtout que le critère de qualité associé à cet axe est bien traduit par une mesure moyenne du paramètre $h^{*}$ sur l'ensemble de la surface du placage.

Nous ne présentons pas l'axe 3 qui est difficile à élucider.

Sens d'utilisation des critères de qualité de l'aspect des placages

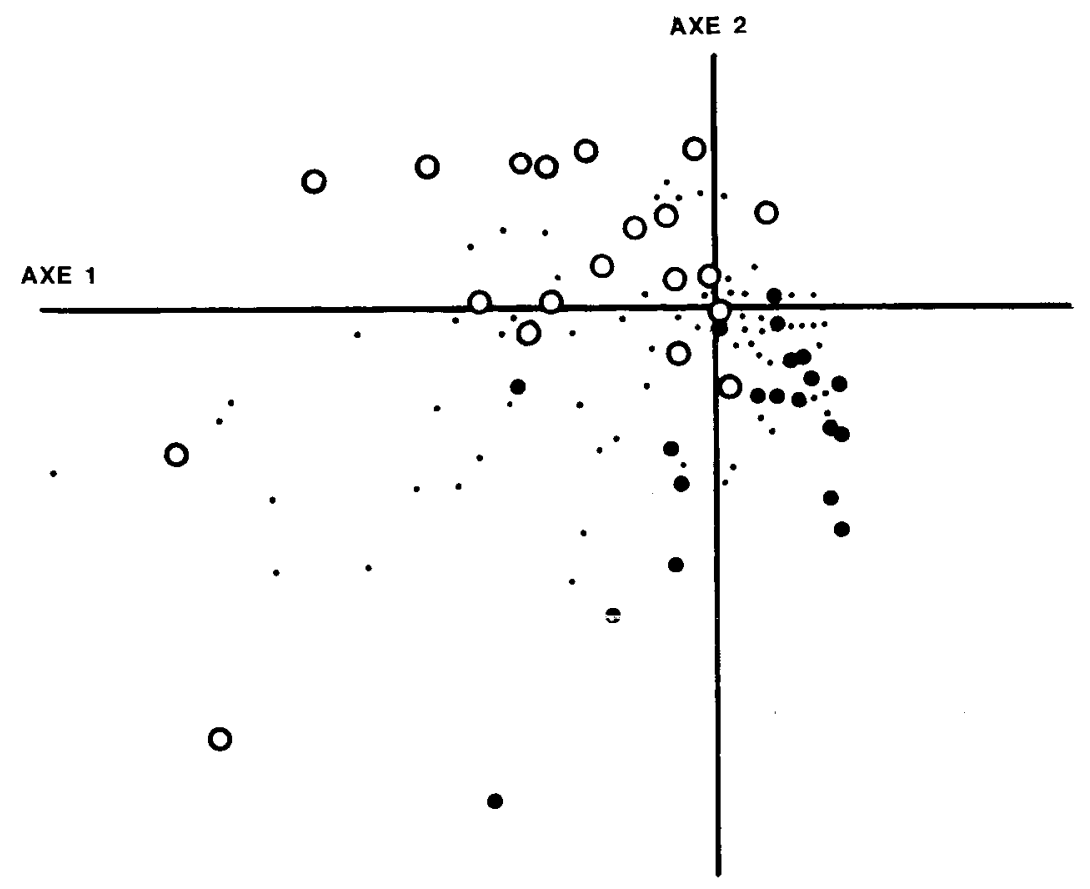

couples de placages avec écart $\Delta h^{*}$ supérieur à 2

$O$ placages avec valeur de $h^{*}$ forte

- placages avec valeur de $h^{*}$ faible

- projections des autres placages

Fig 6. Recherche d'adéquation entre la mesure de $h^{\star}$ et le critère de tonalité dégagé par l'axe 2 (AFC sur les choix en qualité de 90 professionnels du bois aux 53 couples de placages). 


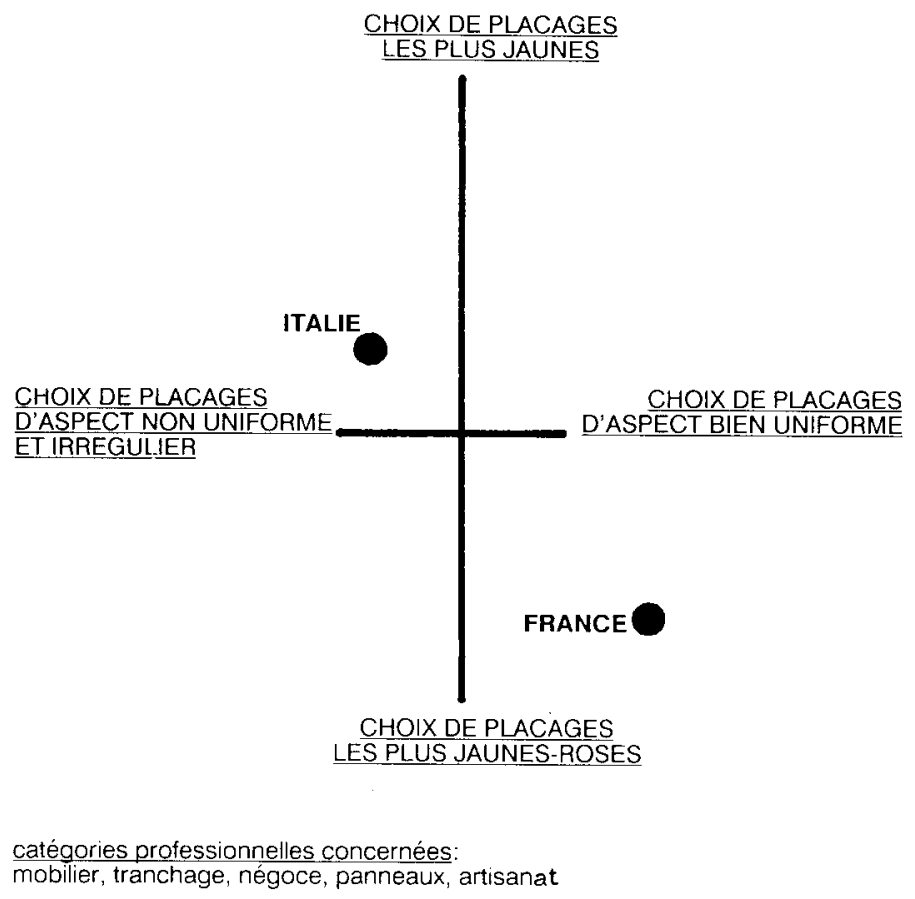

Flg 7. Projection sur le premier plan interprété des points moyens de la variable illustrative pays (Italie et France) (AFC sur les choix en qualité de 90 professionnels du bois aux 53 couples de placages).

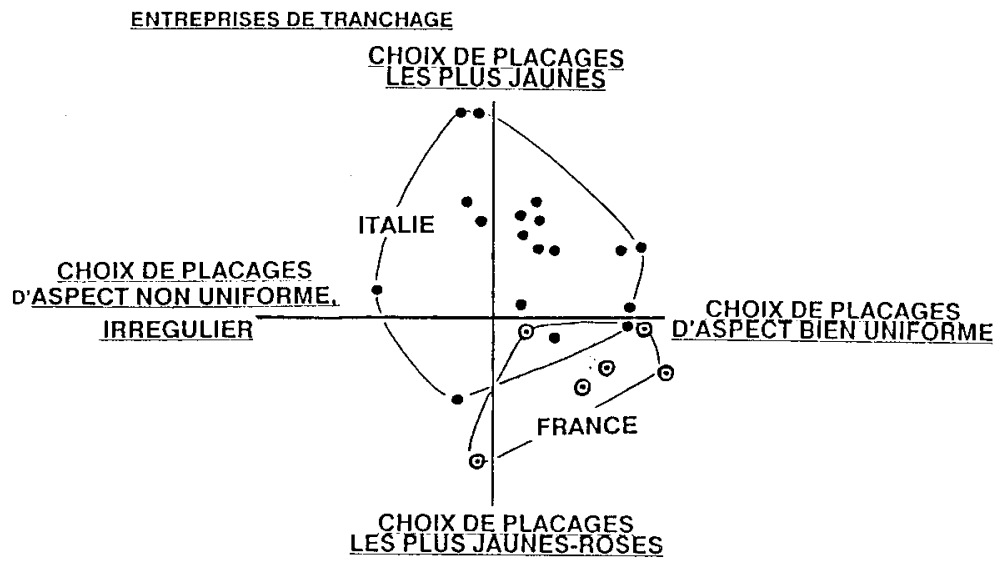

Fig 8. Projection sur le premier plan interprété des individus appartenant à une entreprise de tranchage (AFC sur les choix en qualité de 90 professionnels du bois aux 53 couples de placages). 
Les professionnels français sont moins nombreux que les professionnels italiens qui sont prépondérants dans l'analyse. La projection de leurs points respectifs positionne les premiers par rapport aux seconds quant au sens d'utilisation qu'ils adoptent pour les 2 critères mis en évidence (fig 7) : d'ores et déjà, il apparaît que l'ensemble des professionnels français est attaché a l'aspect uniforme et régulier des placages ainsi qu'à une tonalité de couleur légèrement rosée.

Nous pouvons également observer la plus ou moins bonne cohérence de chacune des catégories professionnelles, en projetant sur ce plan 1-2 les nuages des points individus correspondant à chacune d'elles. Par exemple :

- Les entreprises de tranchage françaises (fig 8) semblent se distinguer de leurs homologues italiennes principalement a travers les tendances exprimées par l'axe 2 : toutes les 2 préfèrent des placages uniformes d'aspect, mais les premieres sont attirées par les plus jaune-rose tandis que les secondes le sont plutôt par les plus jaunes ;

- les entreprises de fabrication de mobilier françaises et italiennes (fig 9) se séparent sur les tendances représentées par l'axe 1. Les Italiens semblent enclins à choisir des placages moins uniformes et réguliers que leurs collègues français. Ils recherchent en fait un aspect plus "vivant» du matériau. Ce sont donc eux qui ont le plus contribué a la création de l'axe 1 de l'AFC;

- les négociants italiens (fig 10) n'apparaissent pas unanimes pour une tendance particulière, contrairement aux français qui affirment nettement leur préférence pour des placages à la fois plus jaune-rosé et plus uniformes d'aspect. Ils rejoignent en cela les trancheurs français.
Rien n'est apparu nettement pour les catégories des artisans, des entreprises d'agencement et des fabricants de panneaux pour lesquelles les nuages des italiens et des français s'interpénétraient et étaient assez dispersés.

Tous ces résultats nous indiquent que les 2 critères dégagés, basés respectivement sur les caractéristiques d'uniformité et de tonalité de la couleur des placages, sont utilisés par des groupes professionnels homogènes, et qu'ils ne sont donc pas le fruit du hasard ou de quelques individus marginaux quant à leurs choix.

\section{Appréciation de l'importance de la clarté des placages}

\section{Présentation}

L'AFC vient de mettre en lumière des critères importants pour la qualité de l'aspect des placages de chêne. Nous avons vu que ces critères sont utilisés de manière différente par les divers groupes professionnels, et c'est précisément à cause de ces oppositions qu'ils ont été mis à jour par l'AFC.

Il semble qu'aucun axe soit en relation avec la clarté des placages. Un tel résultat ne peut manquer de nous intéresser. En effet, nombreux sont les professionnels qui nous ont dit rechercher avant tout des placages bien clairs. Cette absence d'axe pourrait donc être due au choix quasi-unanime du placage le plus clair pour les couples dont les 2 échantillons étaient très differents en clarté.

Pour étudier cela, nous avons procédé à des comptages d'effectifs de réponses particulières.

Au total, sur 4770 réponses (90 professionnels du bois $\times 53$ couples de placages de chêne), 1098 étaient des 


\section{ENTREPRISES DE FABRICATION DE MOBILIER}

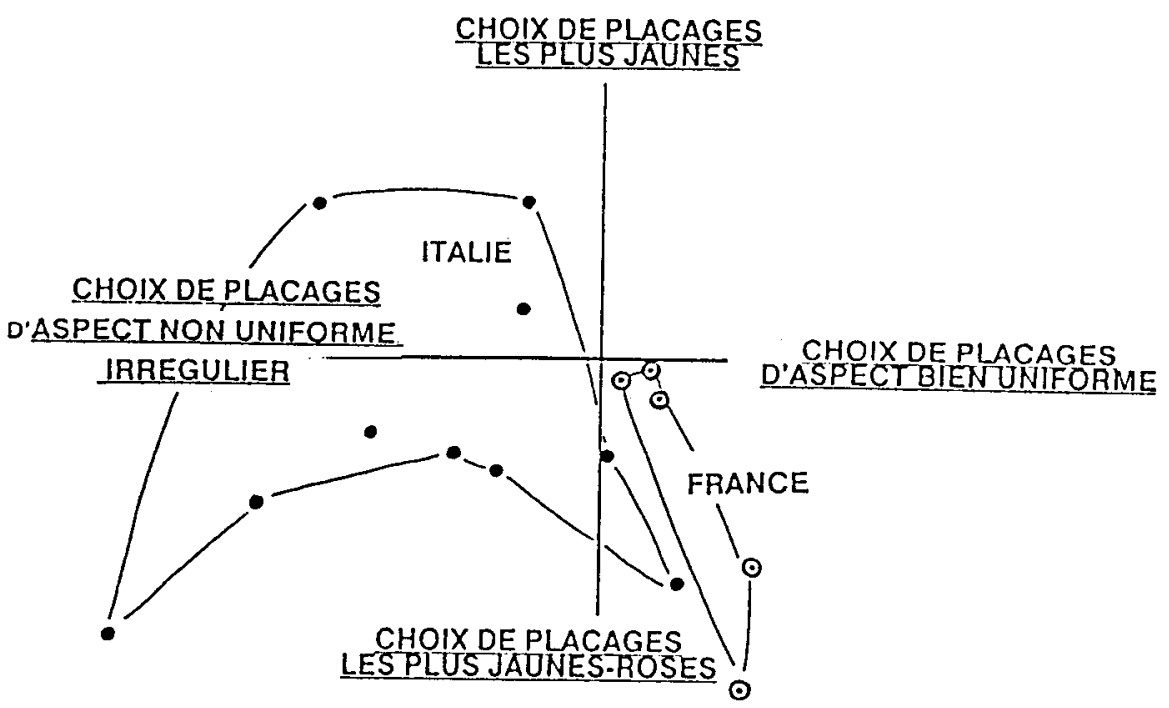

Fig 9. Projection sur le premier plan interprété des individus appartenant à une entreprise de fabrication de mobilier (AFC sur les choix en qualité de 90 professionnels du bois aux 53 couples de placages).

\section{ENTREPRISES DE NEGOCE}

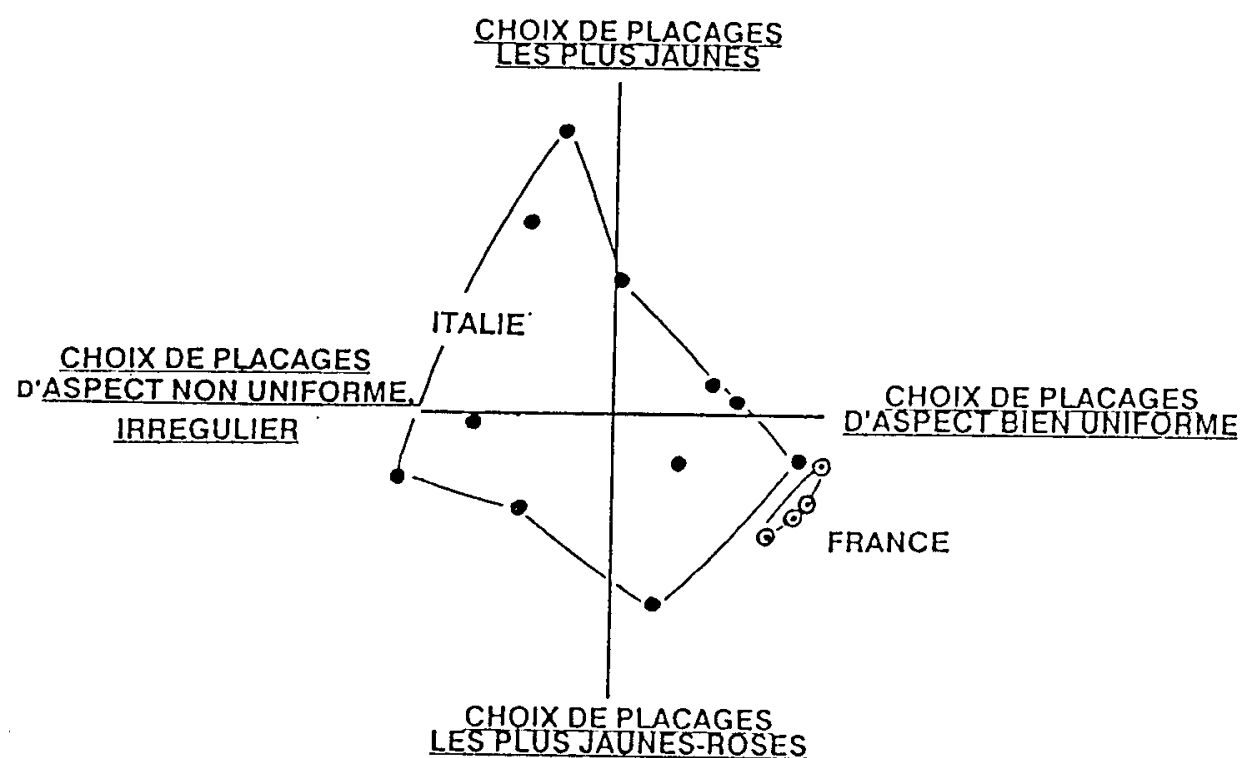

Fig 10. Projection sur le premier plan interprété des individus appartenant à une entreprise de négoce; (AFC sur les choix en qualité de 90 professionnels du bois aux 53 couples de placages). 
non-choix, 2156 choix se sont portés sur les plaques claires (ayant les valeurs de $L^{*}$ les plus élevées par rapport a celles auxquelles elles étaient comparées), et 1516 sur les plus sombres. Donc, d'une manière globale, pour les 53 couples confondus, $59 \%$ des choix proprement dits étaient favorables au placage le plus clair.

Ce résultat global tient compte des réponses qui concernaient aussi bien les couples dont les 2 placages étaient très peu différents en clarté que d'autres pour lesquels la différence était très grande. II est plus judicieux de s'intéresser au nombre de choix du placage le plus clair en fonction de l'écart de clarté entre les 2 placages.

Dans cet esprit, un classement des couples a été réalisé de la même façon qu'à la figure 3 mais cette fois-ci en utilisant l'écart en luminance entre leurs 2 placages pour construire un tableau de contingence (présenté ici

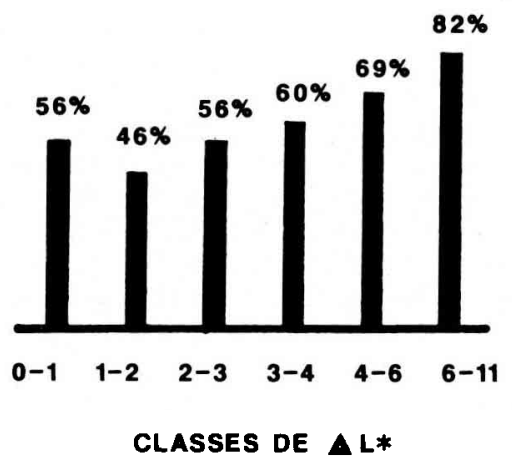

\% DE CHOIX DU PLACAGE LE PLUS CLAIR par rapport aux nombre de choix effectifs (non-choix inclus)

Test du $\chi^{2}$ deux significatif (1\%):

les classes different significativement entres elles

Fig 11. Influence de l'écart en clarté entre deux placages sur le nombre de choix du plus clair; (analyse des choix de 90 professionnels du bois aux 53 couples de placages). sous la forme d'un histogramme, fig 11) qui comptabilise, pour chacune des classes, les effectifs des réponses. Le text du $\chi^{2}$ est significatif et montre que le degré d'écart en clarté des couples conditionne le choix de la plaque la plus claire. L'importance de la clarté des placages pour la qualité est donc bien réelle.

\section{CONCLUSION}

Il a été montré de manière objective lors de cette étude que la couleur des placages de chêne est une composante primordiale de leur qualité.

En effet, apres avoir mis en évidence le rôle de la couleur dans les différents choix en qualité effectués par les professionnels, nous nous sommes attachés à l'analyser de manière plus fine. Ainsi, la clarté des placages est apparue comme une caractéristique essentielle de qualité, recherchée par une majorité d'individus, et bien décrite par la mesure moyenne de la clarté $L^{*}$ (paramètre du système CIELAB 1976 de mesure des couleurs).

Il est également ressorti de nos résultats que l'uniformité d'aspect et la tonalité de la couleur des placages sont 2 caractéristiques importantes dans les choix en qualité, mais prises en compte de manière différente selon les groupes professionnels. Quelles que soient ces divergences, ces 2 critères peuvent être correctement traduits respectivement par les mesures du coefficient de variation de la clarté $s L^{\star} / \mathrm{mL}^{*}$ sur la surface du placage et de l'angle de couleur moyen $h^{\star}$.

La concordance entre les mesures objectives de parametres dans le système CIELAB et l'appréciation professionnelle de la qualité des placages de chêne offre donc la possibilité d'éva- 
luer la qualité potentielle de la production de chêne de qualité tranchage.

Ce résultat important justifie les essais déjà entrepris pour étudier le déterminisme et stationnel de la couleur du bois de chêne (Flot, 1988). Ces études peuvent d'ailleurs être réalisées en utilisant la mesure de la couleur du bois sur les arbres encore vivants, à partir du tranchage industriel de carottes de sondage, et en utilisant les paramètres $L^{*}$ et $h^{\star}$ du système CIELAB (Janin et Mazet, 1987 ; Mazet, 1988).

À ces caractéristiques de couleur, qui font l'objet de cet article, il faut ajouter le dessin du bois sur la surface des placages qui, (nous ne l'avons pas présenté ici), semblait être en relation avec un autre critère professionnel (Mazet, 1989). Le dessin du bois est en relation avec le mode de tranchage des grumes, mais aussi bien sûr avec la largeur des accroissements annuels et le plan ligneux du bois d'une manière générale.

Aussi, une poursuite de ce travail pourrait être axée sur l'étude des plans ligneux de chêne (incluant la largeur des cernes, la texture et autres caractéristiques anatomiques), qui fournissent les figurations du bois les plus appréciées par les professionnels concernés, donc les plus aptes au tranchage. Cela pourrait d'ailleurs être réalisé sur la base d'une autre enquête, dont le protocole serait à définir avec soin en fonction de l'objectif recherché.

\section{REMERCIEMENTS}

Nous tenons à remercier ici l'ensemble des professionnels, français et italiens, artisans et responsables d'entreprises qui ont bien voulu nous recevoir pour participer a cette enquête, et en particulier à la société Ober France de Longeville-en-Barrois (55), qui nous a aimablement fourni de nombreuses feuilles de placages de chene pour la constitution de nos échantillons. Nos remer- ciements vont également a $\mathrm{MM} \mathrm{J} \mathrm{C} \mathrm{Pierrat}$ et $D$ Xeuxet, du CRF de Champenoux pour leurs conseils relatifs au traitements statistique et informatique.

\section{RÉFÉRENCES}

Anonyme (1985) Les échanges extérieurs. Inf Bois, 58, 24-25

Anonyme (1985) Les industries du bois en 1983. - Rev bois, 3, 38-40

Anonyme (1987) Le marché du bois: situation en février 1987. - Inf Bois, 64, 8-12

Bachacou J, Masson JP, Millier C (1981) Manuel de la programmathèque statistique AMANCE 81. INRA, Département de Biométrie, $516 \mathrm{pp}$

Benzecri F, Benzecri JP (1980) L'analyse des correspondances, exposé elémentaire. Dunod, $423 \mathrm{pp}$

Brun-Chaize MC (1978) Le paysage forestier, analyse des criteres de préférences du public a partir de photographies. Cah Anal Donnees, III, 65-78

Flot JL (1988) La couleur du chêne de tranchage français. - Mémoire de $3^{\theta}$ année ENITEF, INRA, centre de recherches forestieres de nancy, station de recherches sur la qualité des bois, $89 \mathrm{pp}$

Janin G (1987) Mesure de la couleur du bois. Intérêt forestier et industriel. - Ann Sci For, 44, 455-472

Janin G, Mazet JF (1987) Mesure de la variabilité de la couleur du bois. Nouvelle méthode appliquée aux carottes de sondage. - Ann Sci For, 44, 119-126

Javeau C (1985) L'enquête par questionnaire : manuel à l'usage du praticien. Univ Bruxelles, Éditions d'Organisation. Paris, $100 \mathrm{pp}$

Kowaliski P (1978) Vision et mesure de la couleur, Masson, Paris, $197 \mathrm{pp}$

Lebart L, Fenelon JP (1973) Statistiques et informatique appliquees. Dunod, $457 \mathrm{pp}$

Mazet JF (1988) Couleur et qualité des placages de chêne et étude de leur comportement photochimique. Thèse de doctorat de l'université de Nacy I, spécialité sciences du bois, $136 \mathrm{pp}$

Mazet JF (1989) Recherche de critères pour l'aspect (dessin et couleur) des placages de bois de chêne. Cah Anal Données, XIV (3) $365-376$ 